\title{
AN INTELLIGENT APPROACH FOR MANAGING MATERIALS PURCHASING IN THE RMC INDUSTRY
}

\author{
Min Wu \\ Dept. of Real Estate and Construction, University of \\ Hong Kong, Pokfulam Road, Hong Kong \\ minwu@hku.hk
}

\author{
Steve Rowlinson \\ Dept. of Real Estate and Construction, University of \\ Hong Kong, Pokfulam Road, Hong Kong \\ steverowlinson@hku.hk
}

\begin{abstract}
A model for comparing the inventory costs of purchasing under the economic order quantity (EOQ) system and the just-intime (JIT) order purchasing system in existing literature concluded that JIT purchasing was virtually always the preferable inventory ordering system especially at high level of annual demand. By expanding the classical EOQ model, this paper shows that it is possible for the EOQ system to be more cost effective than the JIT system once the inventory demand approaches the EOQ-JIT cost indifference point. The intelligent agent paradigm, a natural fit to solve dynamic materials control problem, however has seldom been deployed in construction material management. Based on the EOQ-JIT cost indifference point function, an alternative agent based inventory management system is thus developed. A case study demonstrates how the equation affects the purchasing approach of a material.
\end{abstract}

\section{KEYWORDS}

EOQ, JIT, Multi-agent system, control

\section{BACKGROUND}

The intelligent agent paradigm is a natural fit to certain classes of dynamic materials control problems because the paradigm focuses on coordinating the activities of loosely coupled distributed entities, e.g., various raw materials, materials suppliers, shippers, manufacturers, distribution centers, and retails [1]. One goal of the paradigm is to enable agents to decide when and how much a raw material should be ordered, based on the resources constraints. The existing agent based material control systems are heavily skewed towards just-in-time (JIT) purchasing [2]. This is mainly driven by the success achieved by those JIT companies. By developing a series of mathematical models that directly comparing the cost difference between the EOQ and the JIT purchasing systems, two researchers, i.e. Schniederjans and $\mathrm{Cao}$ [3] concluded that in situations where plants adopting the JIT operations could capitalize 
upon physical plant space square meter reduction, a JIT system would virtually always be preferable to an EOQ system.

However, JIT purchasing is not always successful even if the plants adopting JIT operations can capitalize upon physical plant space reduction [4]. In addition, these two researchers [3] had difficulties to either scientifically or empirically ascertain the capability of an inventory facility to hold the EOQ-JIT cost indifference point's amount of inventory. Their models cannot clearly explain the success achieved by the EOQ companies.

Hence this study has two objectives. The first objective is to revise Schniederjans and Cao's EOQ-JIT cost indifference point function, and to develop a new EOQ-JIT cost indifference point function. The new function will demonstrate that it is still possible for an EOQ system to be more cost effective than a JIT system, even if JIT purchasing may capitalize upon physical plant space reduction. The second objective is, based on the new function, to develop a new agent based material control system. The models of Schniederjans and Cao [3] were developed from the classical EOQ model. Hence, the classical EOQ model should be revisited.

\section{CLASSICAL EOQ MODEL}

Harris's [5] EOQ model, namely, the classical EOQ model, aims to minimize the total of ordering and holding costs, while assuming that some inventory operating costs such as rental, utilities and personnel salary, etc are "fixed" costs. The total annual cost of the classical EOQ system, $T C_{E}$, is the sum of the inventory ordering cost, inventory holding cost, and the cost of the actual purchased units, or:

$T C_{E}=\frac{k D}{Q}+\frac{Q h}{2}+P_{E} D$

where $Q$ is the fixed order quantity, $h$ is the annual cost of holding one unit of inventory in stock, $k$ is the cost of placing an order, $D$ is the annual demand for the item, $P_{E}$ is the purchase price per unit, $D / Q$ is the annual ordering frequency, $Q / 2$ is the annual average inventory level in the inventory facility. It should be noted that although the term "the total annual cost of an inventory item under an EOQ sys- tem" is widely used to refer to " $T C_{E}$ " in Eq. 1, " $T C_{E}$ " is not the actual total annual cost of an inventory item under an EOQ system. The actual total annual cost of an inventory item under an EOQ system should be the sum of " $T C_{E}$ " and the "fixed costs".

"Fixed costs", including "rental, utilities, and personnel salary" were excluded from the inventory holding cost item in Eq. 1. This was also an important assumption made by Schniederjans and Cao [3] when they derived their EOQ-JIT cost indifference points. However, since (a) It is agreed that the so called "fixed costs" were left out from the so called "total annual cost of the EOQ system", and (b) Gaither [6] suggested that the annual inventory holding cost should include the opportunity cost of the working capital tied up in purchased goods, taxes and insurance paid on inventory items, inventory spoilage cost and inventory obsolescence cost, together with the cost of physical storage, and (c) Wantuck [7] proved that the so called "fixed costs" would no longer be constant during JIT operations, and (d) Schniederjans and Cao [2, 3] observed that the saved inventory facilities can be rented out when the annual average inventory level dropped, then there is a reason to include all components of inventory holding costs into the holding cost item, when comparing an EOQ system with a JIT system. To sum up, one of the assumptions of the classical EOQ model, namely, the so called "fixed" costs are excluded from the holding cost item, needs to be revised. Consequently the traditional EOQ model needs to be expanded when comparing an EOQ purchasing system with a JIT purchasing system.

To include the so called fixed cost into the holding cost item, this study assumes that the inventory physical storage costs under the EOQ system, for example, rental, utilities and personnel salaries are linearly related to the average inventory level. However, it should be noted that the inventory physical storage cost under the EOQ system is not necessarily a linear function with the average inventory level. For instance, let us put ourselves in the position of a warehouse man storing cubic boxes. All the boxes are identical. The area of each surface of each box is $\beta m^{2}$. Let us look at a simple scenario of 3 boxes 
and then extend it to a more complicated scenario of $n$ boxes. For 3 boxes, the total inventory space occupied may be $\beta, 2 \beta$ or $3 \beta \mathrm{m}^{2}$. This is shown in Figure 1. For $n$ boxes, the total inventory space occupied may be $\beta, 2 \beta, 3 \beta \ldots(n-1) \beta$, or $n \beta \mathrm{m}^{2}$. This is shown in Figure 2. Hence, the total inventory space taken up by $n$ boxes does not have to be $n \beta m^{2}$, which is the logical extension of the assumption made in this study. Figures 2 shows that this assumption is biased against the EOQ model, as the $n$ boxes may be stored in an inventory space with an area that is smaller than $n \beta m^{2}$.
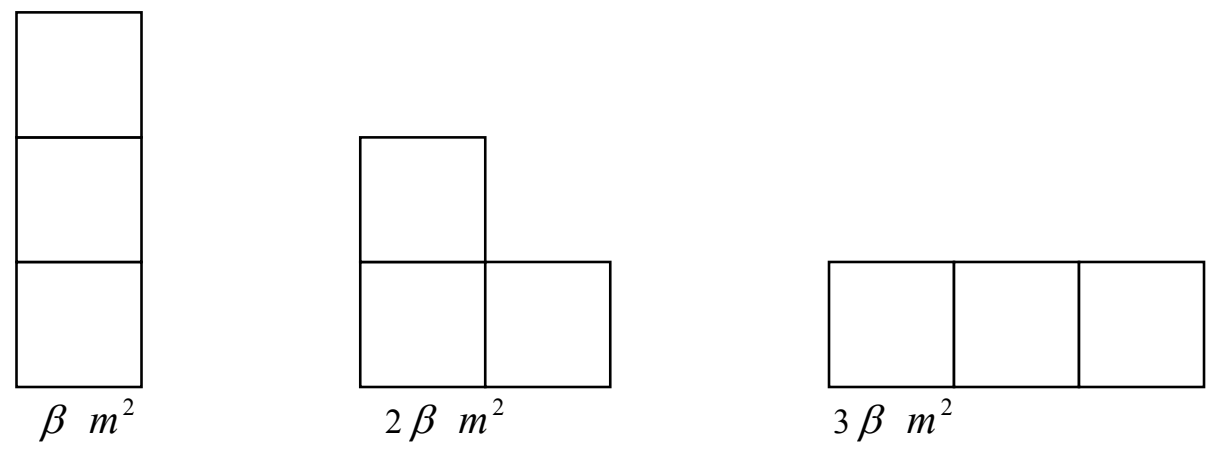

Figure 1. Inventory space occupied by 3 cubic boxes
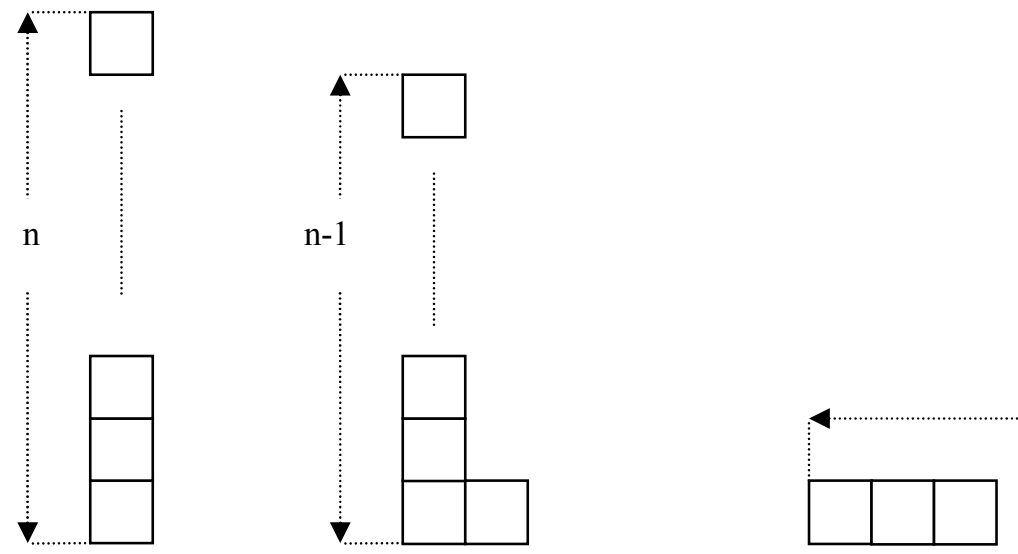

$\mathrm{n}$

$\beta m^{2}$

$2 \beta m^{2}$

$n \beta m^{2}$

Figure 2. Inventory space occupied by $n$ cubic boxes 
In addition, when average inventory level increases, a larger and more compact inventory facility is usually used to save physical plant space. For instance, in places where real estate is costly, it may be prohibitively expensive if not impossible to acquire a large amount of space. This is pertinent to RMC facilities as they necessarily have to be located within travel distance from urban construction sites. In those cases, a larger and more compacted inventory facility is usually used to save physical plant space. It is also important to note that the inventory physical storage costs per unt in a larger sized inventory facilty are usually lower than that in the smaller sized inventory facility, provided that the other conditions, such as rental rate, utility rate and labor rate remain unchanged. The production manager interveiewed by the researchers in a RMC batching plant suggested that if the inventory physical storage costs per unit in the larger sized inventory facility were even higher than that in the smaller inventory facility, then there was no reason to have a larger inventory facility. The company can simply install a few smaller sized inventory facilities together to hold the same amount of inventoy in the RMC industry.

Hence, the assumption made by the authors tended to be quite conservative for the EOQ system when compared with a JIT purchasing system, provided that the annual cost of holding one unit of inventory in stock is determined based on the average value of inventory facilities. This study shows that it is still possible for the EOQ system to be more cost effective than the JIT purchasing system even when a) the JIT operation can experience physical plant space reduction; and b) an unfavorable assumption is made against the EOQ system.

\section{REVISED EOQ-JIT COST INDIFFERENCE POINT}

The total cost under the revised EOQ model is thus:

$T C_{E r}=\frac{k D}{Q}+\frac{H Q}{2}+P_{E} D$

$T C_{E r}$ is the sum of the inventory ordering cost, the expanded inventory holding cost, and the cost of the actual purchased units. $T C_{E r}$ is the actual total cost of the EOQ ordering system and is greater than
$T C_{E r}$ in Eq. 1, as $H$ is significantly greater than " $h$ " in Eq. 1. The optimum order quantity of the revised EOQ model, $Q_{r}^{*}$, derived from Eq. 2 is:

$Q_{r}^{*}=\sqrt{\frac{2 k D}{H}}$

Eq. 4 results in a total annual optimal cost under the EOQ purchasing approach of:

$T C_{E r}=\sqrt{2 k D H}+P_{E} D$

Under the JIT system, the ordering cost and holding cost, including the so called "fix costs" are mainly transferred to the supplier. The total annual cost under the JIT system, $T C_{J}$, thus, is the annual purchase cost [3], given by:

$T C_{J}=P_{J} D$

where $P_{J}$ is the unit price under the JIT system. $P_{J}$ is greater than $P_{E}$. This is to partially reflect the holding costs and ordering costs that have been transferred to the materials suppliers [3]. The cost difference between an EOQ purchasing system and a JIT purchasing system, $Z_{r}$ thus is:

$Z_{r}=\sqrt{2 k D H}+P_{E} D-P_{J} D$

Setting $Z_{r}$ equal to zero, the root of Eq. 7 is the revised EOQ-JIT cost indifference point, $D_{i n d r}$ :

$D_{\text {indr }}=\frac{2 k H}{\left(P_{J}-P_{E}\right)^{2}}$

\section{A NEW AGENT BASED MATERIAL CONTROL SYSTEM}

In this study, an agent system, which based on the newly developed EOQ-JIT cost indifference point function, is developed to decide whether a material should be ordered by using EOQ approach or JIT approach. Fig. 3, Fig. 4 shows the scenario of the proposed multi-agent system for material control.

The system proposed in Fig. 3 consists of three agents, namely material agent, logistics manager agent, and production manager. The material agent records the historical data of each of the material 
regarding its ordering cost, holding cost, ordering frequency, its supplier information etc. The logistics manager agent, based on the EOQ-JIT cost indiffer- ence point, calculates the EOQ-JIT cost indifference point and decides whether a material should be purchased via an EOQ or JIT

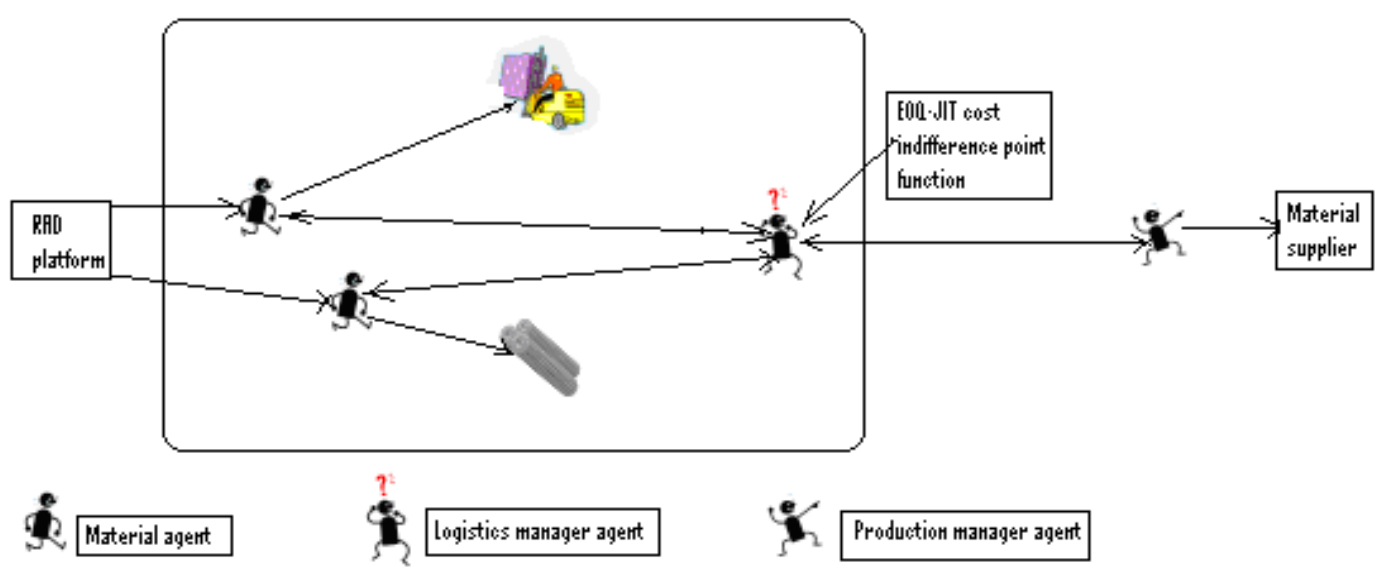

Figure 3 Scenario of the model

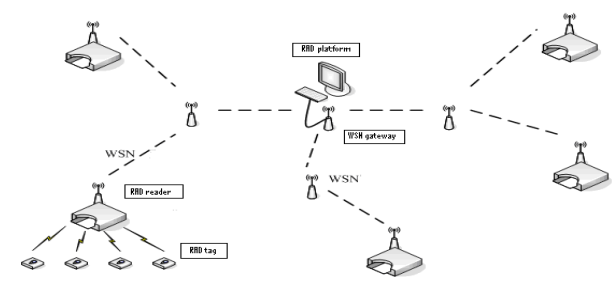

Figure 4. RFID platform

\section{A CASE STUDY}

This case study demonstrates how the EOQ-JIT cost indifference point function may affect the purchasing approach of a material.

Ready mixed concrete (RMC) is a product that is widely used in the construction of building and civil works in the construction industry. The production of $\mathrm{RMC}$ is a highly repetitive manufacturing process [3]. Cement, one of the raw materials of RMC may be purchased either using the EOQ system or the JIT system [4]. In Singapore, most of the cement consumed was imported mainly from Japan by 40,000- ton cement carriers. The cost of placing an order for 40,000 cement carrier from Japan was $k=\mathrm{S} \$ 432$, $000 /$ order for transportation alone. The annual cost of holding one ton of cement was $H=\mathrm{S} \$ 344$ /year per ton. Purchasing cement according to the EOQ model costs $P_{E}=\mathrm{S} \$ 40 /$ ton. If cement was purchased under a JIT system, the cost was $P_{J}=\mathrm{S} \$ 65$ /ton. The data for this case study were collected by interviewing the overseas investment manager, the financial manager, the production manager and the customer service supervisor of the cement division of a supplier. Based on Eq. 3, the economic order quantity was $Q_{r}^{*}=36,139$ ton / order, which was close to the routine order quantity 40,000 ton / order. Hence, Eq. 7 can be used to derive the EOQ-JIT cost indifference point. According to Eq. 7, $D_{\text {indr }}$ the EOQ-JIT cost indifference point was 475,545 ton. A survey revealed that the RMC suppliers in Singapore, whose annual cement demand were greater than $D_{\text {indr }}$ purchased their cement in an EOQ fashion [4]. 


\section{CONCLUSIONS}

The existing agent based inventory management approaches are heavily skewed toward just-in-time (JIT) purchasing. This paper develops an EOQ-JIT cost indifference point function, and shows that it is possible for the EOQ system to be more cost effective than the JIT system. Based on the function, an alternative agent based inventory management system is developed. A case study demonstrates how the equation affects the purchasing approach of a material.

\section{REFERENCE}

[1] Wagner T., Guralnik V., and Phelps J., proceedings of "Software agents: Enabling dynamic supply chain management for a build to order product line," Agents in Business Automation, pp.1-9, 2002.

[2] Yung S.K., Yang C.C., Lau A.S.M., and Yen J., “Applying multi-agent technology to supply chain management," Journal of Electronic Commerce Research, 1(4), pp.119-132, 2000.

[3] Schniederjans M.J. and Cao Q., "An alternative analysis of inventory costs of JIT and EOQ purchasing," International Journal of Physical Distribution \& Logistics Management, 31 (2), pp.109 - 123, 2001.

[4] Wu M., Modeling JIT purchasing in the ready mixed concrete industry. Unpublished $\mathrm{PhD}$ thesis, the $\mathrm{Na}-$ tional University of Singapore, 2004.

[5] Harris F.W., Operations and Cost - Factory Management Series, A.W. Shaw Co, Chicago, 1915.

[6] Gaither N., Production and Operations Management, Duxbury Press, Belmont, CA. 1996.

[7] Wantuck K.A., Just-In-Time for America: a Common Sense Production Strategy, The Forum Ltd., Milwaukee, WI, 1989. 